\title{
HIGH-SPEED LIQUID CHROMATOGRAPHY OF SUGAR ACIDS ON AN- ION-EXCHANGE RESINS
}

\author{
P. J. M. DIJKGRAAF, L. A. Th. VERHAAR*, W. P. T. GROENLAND and K. VAN DER WIELE \\ Eindhoven University of Technology, Department of Chemical Technology, P.O. Box 513, 5600 MB Eind- \\ hoven (The Netherlands)
}

(First received February 13th, 1985; revised manuscript received March 20th, 1985)

\section{SUMMARY}

Mixtures of aldonic and aldaric acids are difficult to analyse. Aldonic acids can be separated in a satisfactory way on anion-exchange resins with a solution of sodium chloride as eluent. Under these circumstances, however, aldaric acids show unacceptably long elution times and peak broadening. The ability of aldaric acids to form non-adsorbable complexes with magnesium ions lowers their elution times, particularly of the low-molecular-weight acids, by up to 25 fold. This effect can be controlled by adjusting the magnesium ion concentration in the eluent. It is thus possible to achieve rapid, accurate analysis of mixtures of aldonic and aldaric acids by a proper choice of the magnesium and chloride ion concentrations in the eluent.

\section{INTRODUCTION}

Sugar acids are an alternative to polyphosphates in detergents. They can be produced by oxidation of glucose with commercially available platinum on carbon catalysts. Usually a mixture is obtained containing several aldonic and aldaric acids. To study this reaction a fast and accurate method of analysis is important.

Much is known about the chromatographic analysis of mixtures of aliphatic and aromatic carboxylic acids; good reviews have been written by Jandera and Churáček $^{1}$ and Schwarzenbach ${ }^{2}$. Despite the large amount of work on this subject, however, very little has been published about the analysis of a special group of carboxylic acids: the sugar acids.

Sugar acid molecules contain many hydroxyl groups, which means that the molecule lacks a non-polar region. For this reason, paired-ion chromatography and reversed-phase chromatography are not suitable methods for analysing solutions of these compounds. Methods based on the use of pure silica cannot be used either, because of the strong interaction of the sugar acids with this solid phase.

Much research has been done on the analysis of solutions of aldonic acids and alduronic acids with anion-exchange resins and acetic acid-sodium acetate solutions as eluent ${ }^{3-6}$. Anion-exchange resins in the formate form are also used to analyse mixtures of carboxylic acids in food products ${ }^{7-9}$. The analysis of gluconic acid and 
three types of ketogluconic acid with an anion-exchange resin and a solution of ammonium formate as eluent has been described by Blake et al. ${ }^{10}$. In combination with anion-exchange resins, borate ${ }^{11,12}$, phosphate ${ }^{13,14}$, and sulphate media ${ }^{15,16}$ have also been used as eluents.

During the adsorption of carboxylic acids on anion-exchange resins, both ionexchange adsorption and molecular adsorption are generally involved. The amount of counter ions present in the eluted volume is a measure of the ion-exchange adsorption ${ }^{1}$, and the degree of molecular adsorption can be calculated from the difference between the overall adsorption and the ion-exchange adsorption. The elution time of (sugar) acids with equal $\mathrm{p} K_{z}$ values can be influenced by the percentage of cross-linking of the resin used; compounds of a high molecular weight normally elute faster than smaller ones.

Until now only the analysis of solutions of aldonic acids, of aldaric acids and of mixtures of aldonic and alduronic acids have been described in the literature. Dirkx and Verhaar ${ }^{15}$ reported the analysis of mixtures of aldonic and aldaric acids with sodium sulphate as eluent, but the separation of the aldonic acids was not satisfactory.

This paper describes a method of analysis for mixtures of aldonic and aldaric acids which makes use of the ability of the aldaric acids, particularly those of lower molecular weight, to form complexes with cations. In this way short elution times for aldonic and aldaric acids are achieved.

\section{EXPERIMENTAL}

\section{Apparatus and procedure}

A Spectra Physics type SP 8100 liquid chromatograph was used together with a variable-wavelength UV-VIS detector, type SP 8400 , and a data system, type SP 4100 .

The separations were carried out on a $250 \times 4.6 \mathrm{~mm}$ I.D. Lichroma SS column, slurry packed with BA-X8 resin (Alltech), particle size 7-10 $\mu \mathrm{m}$, at a temperature of $85^{\circ} \mathrm{C}$. Unless stated otherwise the flow-rate was $1.0 \mathrm{ml} / \mathrm{min}$ and the pressure drop over the column was $4.5 \mathrm{mPa}$. The eluent was mixed by the solvent-delivery system from a solution of sodium chloride $(0.2 M)$ and magnesium chloride $(0.1 M)$ and water. These solutions, water and samples were filtered over a Millipore type MAWP filter $(0.45 \mu \mathrm{m})$ and, exccpt for the samples, wcrc degassed by the helium method. The injection volume was $10 \mu \mathrm{l}$ and the detection wavelength was $212 \mathrm{~nm}$.

Mixtures of aldaric acids, of aldonic acids and of aldonic and aldaric acids, containing each compound at a concentration of $50 \mathrm{mmol} / \mathrm{l}$, were used to study the elution times of these compounds as a function of the eluent composition. Therefore several analyses were made with eluents of constant concentrations of chloride and magnesium ions. These sample solutions were brought to $\mathrm{pH} 9$ to prevent formation of lactones.

\section{Reagents}

Glyoxylic acid, sodium gluconate, tartaric acid, glucaric acid and tartronic acid were purchased from Janssen Chemica, disodium oxalate and sodium formate from Merck, glycolic acid from Fluka, potassium arabonate from Hicol B.V. and sodium bicarbonate from $J$. T. Baker. 


\section{RESULTS AND DISCUSSION}

It is known that in ion-exchange chromatography the elution time of a compound depends, among other things, on the concentration of counter ions in the eluent. For our system this is shown in Figs. 1 and 2. In case of both the aldonic and aldaric acids it appears that acids of low molecular weight are influenced more strongly by the chloride concentration than acids of high molecular weight. Figs. 1 and 2 also show that aldaric acids are held more strongly than the aldonic acids (apparently by the double negative charge of these acids), and that the elution times of the aldaric acids are more sensitive to the chloride ion concentration in the eluent.

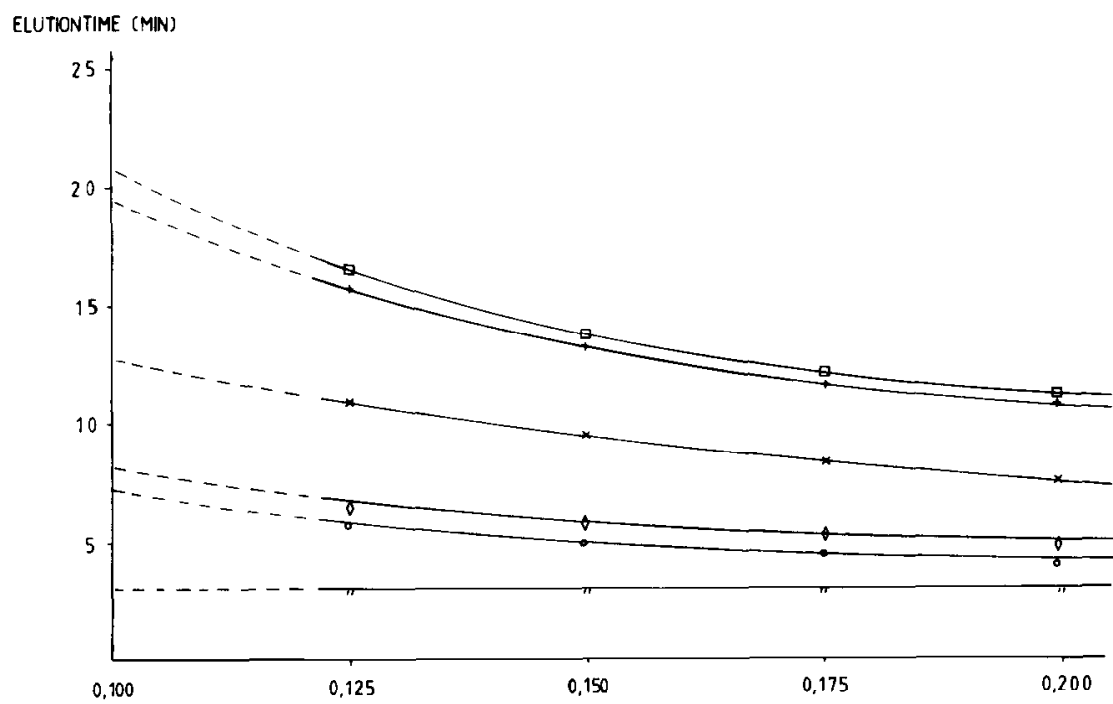

$\left.\left[\mathrm{Cl}^{-}\right] \mathrm{CMO} / \mathrm{D}\right]$

Fig. 1. Influence of the chloride ion concentration on the elution times of the aldonic acids. For experimental conditions see Apparatus and procedure. Data points: $\square=$ formic acid; $+=$ glyoxylic acid; $x=$ glycolic acid; $O=$ arabinonic acid; $O=$ gluconic acid; $\|=$ sodium bicarbonate.

The elution times of the aldonic acids are influenced to a minor extent by the presence in the eluent of magnesium chloride, which acts as a complex-forming agent. When the elution time of an aldonic acid is plotted as a function of $\left[\mathrm{Mg}^{2+}\right]$ at a constant chloride ion concentration, a straight line is obtained with a small slope. Table I lists the slopes of these lines for the aldonic acids at several chloride ion concentrations. It can be seen that as the chloride ion concentration is increased, the slopes of the lines become smaller. This effect is more pronounced for aldonic acids of low molecular weight.

It is possible to calculate the elution time of an aldonic acid at certain concentrations of chloride and magnesium ions by combining the results of Fig. 1 and Table 1. Although the magnesium ion concentration in the eluent is of minor importance for aldonic acids, this is not the case for aldaric acids. Fig. 3 shows the elution times of the aldaric acids plotted as a function of the magnesium ion concentration at a constant chloride ion concentration of $0.125 \mathrm{M}$, and it is clear that the elution times of the low-molecular-weight aldaric acids in particular can be dras- 


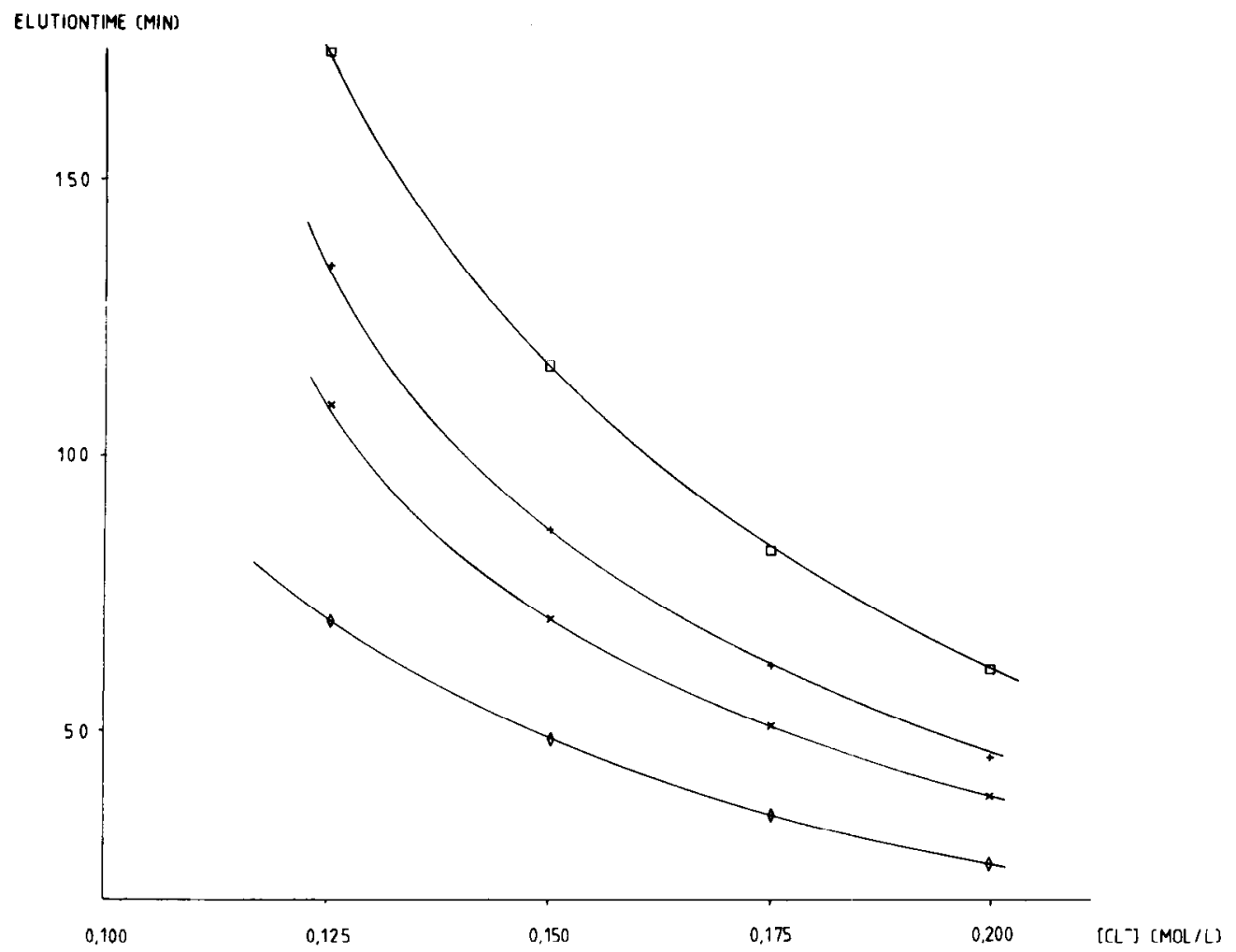

Fig. 2. Influence of the chloride ion concentration on the elution times of the aldaric acids. For experimental conditions see Apparatus and procedure. Data points: $\square=$ oxalic acid; $+=$ tartronic acid; $x=$ tartaric acid; $\diamond=$ glucaric acid.

tically reduced by applying a relatively high magnesium ion concentration. These results are in agreement with the results of Lee and Samuelson ${ }^{17}$ and Bengtsson and Samuelson ${ }^{18}$, who analysed mixtures of aldaric acids with pure magnesium acetate solutions as eluent. From the work of Cannan and Kibrick ${ }^{19}$, it appears that $\alpha$ -

\section{TABLE I}

SLOPES OF THE STRAIGHT LINES FOUND WHEN THE ELUTION TIME OF AN ALDONIC ACID IS PLOTTED AGAINST THE MAGNESIUM ION CONCENTRATION, AT DIFFERENT CHLORIDE ION CONCENTRATIONS

Slope values in $\min 1 / \mathrm{mmol}$.

\begin{tabular}{lllll}
\hline Compound & \multicolumn{2}{l}{$\left.l \mathrm{Cl}^{-}\right\rfloor(\mathrm{mol} / \mathrm{l})$} & & \\
\cline { 2 - 5 } & 0.125 & 0.150 & 0.175 & 0.200 \\
\hline Formic acid & 0.0325 & 0.0245 & 0.0225 & 0.0175 \\
Glyoxylic acid & 0.0300 & 0.0285 & 0.0250 & 0.0220 \\
Glycolic acid & 0.0400 & 0.0350 & 0.0275 & 0.0200 \\
Arabinonic acid & 0.0163 & 0.0145 & 0.0100 & 0.0093 \\
Gluconic acid & 0.0138 & 0.0135 & 0.0125 & 0.0100
\end{tabular}




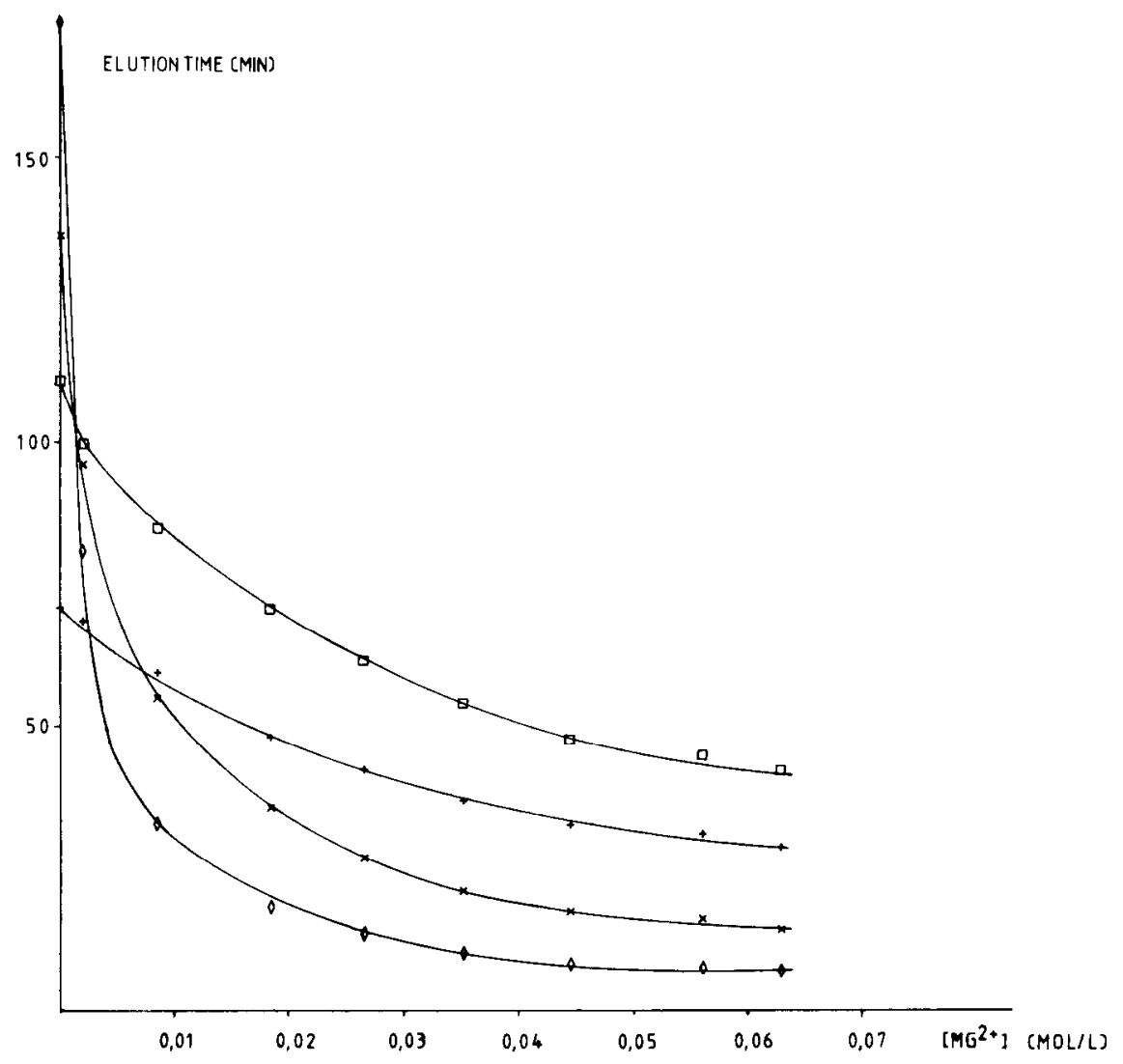

Fig. 3. Influence of the magnesium ion concentration on the elution times of aldaric acids at a chloride ion concentration in the eluent of $0.125 \mathrm{M}$. For experimental conditions see Apparatus and procedure. Data points: $\diamond=$ oxalic acid; $x=$ tartronic acid; $\square=$ tartaric acid; $+=$ glucaric acid.

hydroxyl groups play an important role in complex formation between sugar acids and magnesium ions. This in contradiction to the remaining part of the molecule. The aldaric acids used form stronger complexes than the aldonic acids because of the presence of two carboxylic and $\alpha$-hydroxyl groups. This complex formation leads to compounds that are less adsorbable and results in low elution times if the eluent contains magnesium ions. Because of the relativcly small influence of the magnesium ion concentration on the elution times of the aldonic acids, it is possible to shift the peaks of the aldaric acids in the chromatogram over great distances with respect to the peaks of the aldonic acids. In this way the analysis of a mixture of aldonic and aldaric acids can be optimized to get the best separation of these compounds in a short time, by choosing the right proportions of the sodium chloride and magnesium chloride in the eluent.

An alternative method has been proposed by Smits ${ }^{20}$, who used two separated solutions of sodium chloride and magnesium chloride. During the analysis of a mixture of aldonic and aldaric acids the eluent was switched from a sodium chloride to a magnesium chloride solution after all aldonic acids had been eluted: a typical chro- 


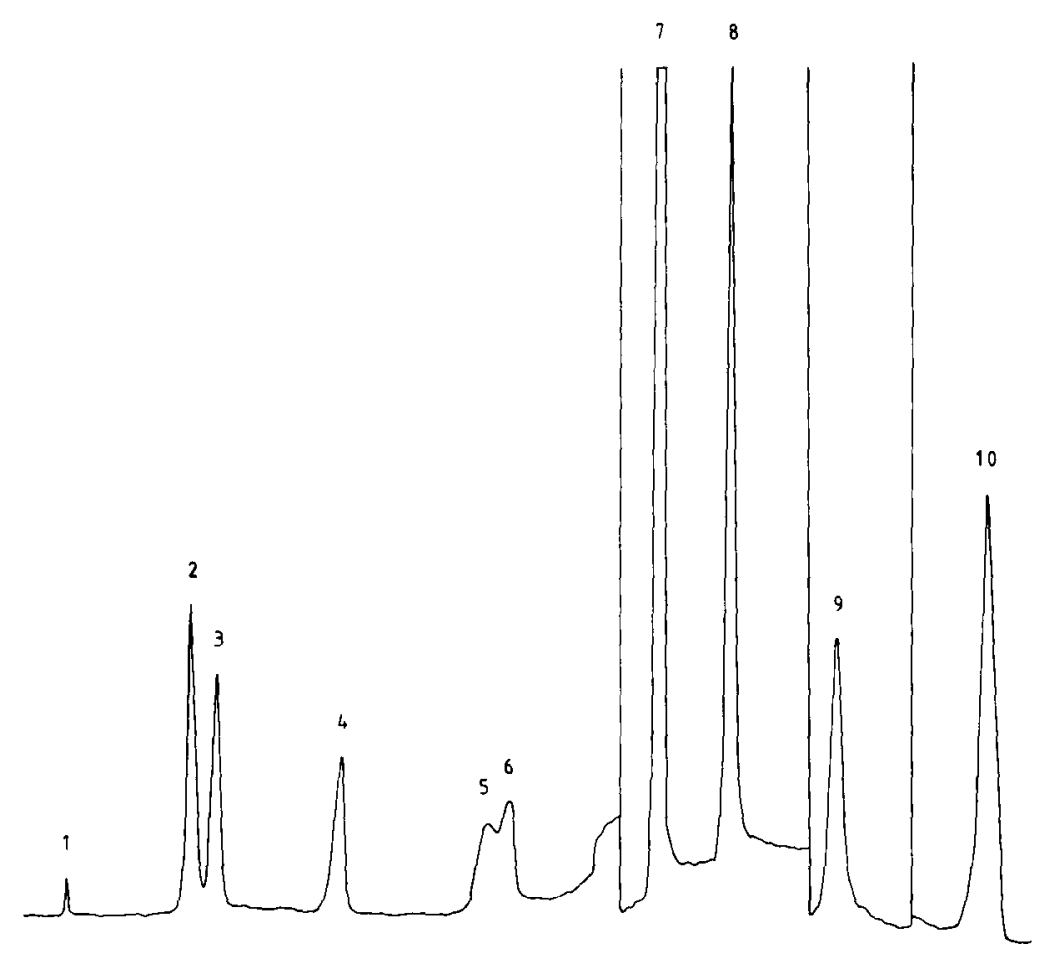

$\begin{array}{llllllll}0 & 5 & 10 & 15 & 20 & 25 & 30 & 35\end{array}$ TIME (MIN)

Fig. 4. Analysis of the mixture of aldonic and aldaric acids used by the procedure used by Smits $^{20}$. The eluent was switched from a $0.125 \mathrm{M}$ sodium chloride to $0.0875 \mathrm{M}$ magnesium chloride at $t=20.1 \mathrm{~min}$. Peaks: 1 = bicarbonate; 2 = gluconic acid; 3 = arabinonic acid; $4=$ glycolic acid; $5=$ glyoxylic acid; $6=$ formic acid; $7=$ oxalic acid; $8=$ tartronic acid; $9=$ glucaric acid; $10=$ tartaric acid.

matogram of the mixture used in this study is given in Fig. 4. Fig. 5 shows a chromatogram of the same mixture, where an eluent was optimized to produce a good separation in a short time. This is done by using an optimal proportion of the sodium chloride and magnesium chloride concentrations as described in this paper. Relatively broad peaks are found for the aldaric acids, probably because of equilibrium reactions between the aldaric acids and the magnesium ions, as described by Verhaar ${ }^{21}$.

In this study, solutions of aldaric acids, of aldonic acids and of aldaric and aldonic acids were used to examine the mutual influence of these compounds on their elution times, and their response during detection. Deviations of up to $3 \%$ in elution times were found. During duplicate experiments, deviations in response during detection were found of the order of the measuring error. The response during detection for sodium bicarbonate is very low. 


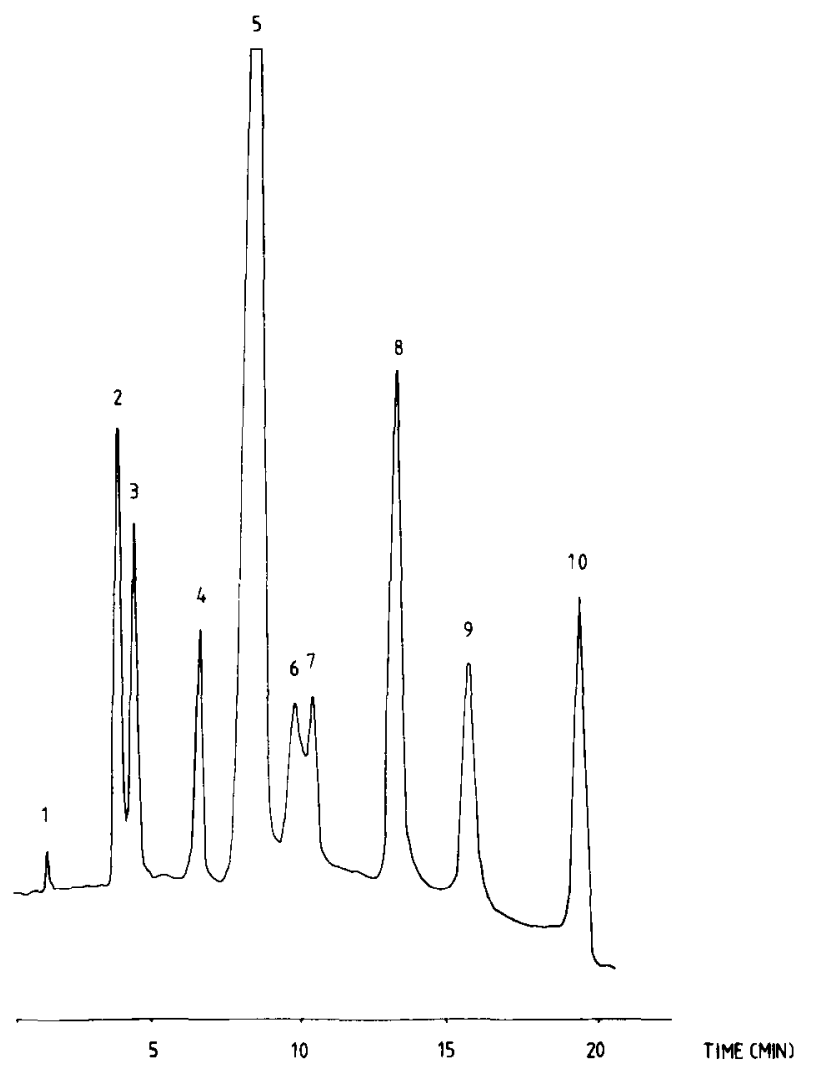

Fig. 5. Optimal chromatogram of the mixture of aldonic and aldaric acids used. As eluent a solution of magnesium chloride $(0.02 \mathrm{M})$ and sodium chloride $(0.16 \mathrm{M})$ was used. The eluent flow-rate was up to 2.0 $\mathrm{ml} / \mathrm{min}$; for remaining experimental conditions see Apparatus and procedure. Peaks $1=$ bicarbonate; $2=$ gluconic acid; $3=$ arabinonic acid; $4=$ glycolic acid; $5=$ oxalic acid; $6=$ glyoxylic acid; $7=$ formic acid; $8=$ tartronic acid; $9=$ glucaric acid; $10=$ tartaric acid.

\section{REFERENCES}

1 P. Jandera and J. Churáček, J. Chromatogr., 86 (1973) 351.

2 R. Schwarzenbach, J. Chromatogr., 251 (1982) 339.

3 B. Alfredsson, S. Bergdahl and O. Samuelson, Anal. Chim. Acta, 28 (1963) 371.

4 U.-B. Larsson, I. Norstedt and O. Samuelson, J. Chromatogr., 22 (1966) 102.

5 B. Carlsson, T. Isaksson and O. Samuelson, Anal. Chim. Acta, 43 (1968) 47.

6 E. Martinsson and O. Samuelson, Chromatographia, 3 (1970) 405.

7 C. W. Davies, R. D. Hartley and G. J. Lawson, J. Chromatogr., 18 (1965) 47.

8 J. K. Palmer and D. M. List, J. Agr. Food Chem., 21 (1973) 903.

9 P. Symonds, Ann. Nutr. Alim., 32 (1978) 957.

10 J. D. Blake, M. L. Clarke and G. N. Richards, J. Chromatogr., 312 (1984) 211.

11 K. Larsson, L. Olsson and O. Samuelson, Carbohyd. Res., 38 (1974) 1.

12 S. Honda, S. Suzuki, M. Takahashi, K. Kaheki and S. Ganno, Anal. Biochem., 134 (1983) 34.

13 L. Bengtsson and O. Samuelson, Anal. Chim. Acta, 57 (1971) 93.

14 L. Bengtsson and O. Samuelson, Chromatographia, 4 (1971) 142.

15 J. M. H. Dirkx and L. A. Th. Verhaar, Carbohyd. Res., 73 (1979) 287.

16 L. Bengtsson and O. Samuelson, J. Chromatogr., 61 (1971) 101. 
17 K. S. Lee and O. Samuelson, Anal. Chim. Acta, 37 (1967) 359

18 L. Bengtsson and O. Samuelson, Anal. Chim. Acta, 44 (1969) 217.

19 R. K. Cannan and A. Kibrick, J. Amer. Chem. Soc.,, 60 (1938) 2314.

20 P. C. C. Smits, Ph.D. Thesis, Eindhoven University of Technology, 1984.

21 L. A. Th. Verhaar, Starch/Stärke, 34 (1982) nr. 10 S 351. 